Experimental basis for laser-plasma interactions in ignition hohlraums at the National Ignition Facility

D. H. Froula, L. Divol, R. A. London, R. L. Berger, T. Doeppner, N. B. Meezan, J. Ralph, J. S. Ross, L. J. Suter, S. H. Glenzer

November 19, 2009

Physics of Plasmas 
This document was prepared as an account of work sponsored by an agency of the United States government. Neither the United States government nor Lawrence Livermore National Security, LLC, nor any of their employees makes any warranty, expressed or implied, or assumes any legal liability or responsibility for the accuracy, completeness, or usefulness of any information, apparatus, product, or process disclosed, or represents that its use would not infringe privately owned rights. Reference herein to any specific commercial product, process, or service by trade name, trademark, manufacturer, or otherwise does not necessarily constitute or imply its endorsement, recommendation, or favoring by the United States government or Lawrence Livermore National Security, LLC. The views and opinions of authors expressed herein do not necessarily state or reflect those of the United States government or Lawrence Livermore National Security, LLC, and shall not be used for advertising or product endorsement purposes. 


\title{
Experimental basis for laser-plasma interactions in ignition hohlraums at the National Ignition Facility
}

\author{
D. H. Froula ${ }^{1}{ }^{*}$ L. Divol, R. A. London, R. L. Berger, T. Doeppner, \\ N. B. Meezan, J. Ralph, J. S. Ross, L. J. Suter, and S. H. Glenzer \\ 1 L-399, Lawrence Livermore National Laboratory, P.O. Box 808, Livermore, CA 94551, USA
}

(Dated: November 18, 2009)

\begin{abstract}
A series of laser plasma interaction experiments at OMEGA (LLE, Rochester) using gas-filled hohlraums shed light on the behavior of stimulated Raman scattering and stimulated Brillouin scattering at various plasma conditions encountered in indirect drive ignition designs. We present detailed experimental results that quantify the density, temperature, and intensity thresholds for both of these instabilities. In addition to controlling plasma parameters, the National Ignition Campaign relies on optical beam smoothing techniques to mitigate backscatter. We show that polarization smoothing is effective at controlling backscatter. These results provide an experimental basis for forthcoming experiments on National Ignition Facility.
\end{abstract}

\section{INTRODUCTION}

Numerous laser-plasma instability studies over the last fifteen years have tried to emulate the electron density and temperature that will be achieved in indirect drive fusion targets at the National Ignition Facility [1]. Generally, these studies have shown strong sensitivity of laserplasma interactions to the exact plasma conditions and laser beam smoothing techniques were shown to lower backscatter levels primarily through the mitigation of filamentation [2-4].

In preparation for indirect drive ignition experiments, we have performed a series of detailed experiments at the Omega laser facility (LLE/U. Rochester) [5] over the last 4 years to better quantify the importance of plasma conditions, plasma length, and beam smoothing techniques on backscatter in a regime relevant to the target designs for the National Ignition Campaign currently underway at Lawrence Livermore National Laboratory. By using a closed geometry (gas-filled hohlraum) and through careful radiation hydrodynamic designs, validated by Thomson scattering measurements, we obtained uniform multimillimeter long plasmas at temperatures between 1.5 and $3.5 \mathrm{keV}$ and densities from 5 to $13 \% n_{c r}$ where $n_{c r}$ is the critical density for 0.35 micron light. We performed detailed measurements of the backscatter from a $0.35 \mathrm{mi}-$ cron interaction beam propagating through these plasmas to obtain density, temperature, and intensity thresholds for both stimulated Raman (SRS) and stimulated Brillouin scattering (SBS). We have also quantified the impact of phase plates and polarization smoothing on these intensity thresholds.

This set of data provides strong guidance for the design of ignition hohlraums and the choice of laser parameters. The key results can be summarized, in a simplified way, as: (1) to insure propagation of 0.351 micron light through millimeters of plasma, keep electron tem-

*Electronic address: froula1@llnl.gov

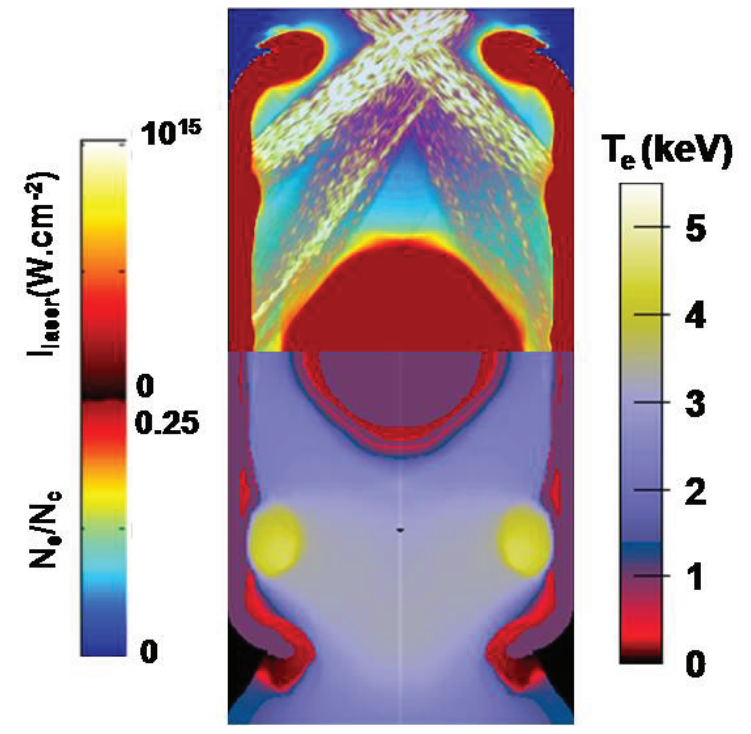

FIG. 1: A 1.2-cm long indirect drive ignition hohlraum is shown with the simulated plasma densities (top), and temperatures (bottom). The laser intensities are superimposed of the top.

perature above $2.5 \mathrm{keV}$, (2) to control SRS in the 10$15 \%$ critical regime, limit the laser intensity to less than $10^{15} \mathrm{~W}-\mathrm{cm}^{-2}$, (3) use polarization smoothing. This will ensure that the linear gains for SBS and SRS remain low and that high intensity speckles present in the laser spots do not create large backscatter. Figure 1 shows the density, temperature, and intensities for an indirect drive fusion design. Backscatter is mitigated by remaining in the strongly damped regime when the laser beam intensity is high and keeping the laser intensity below the backscatter thresholds where the density is high and the temperature is low. In addition to the intrinsic value of the experimental results presented in this paper, the relative success in modeling absolute backscatter levels for a wide variety of parameters has built confidence in the extrapolation to NIF experiments.

In Sec. II, the experimental setup is presented, includ- 
ing a description of the variations in the target platform which has allowed us to systematically study various indirect drive plasma conditions along the propagation path of the NIF inner beams. Section III describes experiments that emulate the high density regime $\left(>10 \% n_{c r}\right)$ created by the expanding gold wall and the blow off plasma from the capsule. In Section IV, experiments are presented that study the middle density regime $\left(8.5 \% n_{c r}\right)$ where there is a potential competition between SRS and SBS. Section V describes experiments to study the laser entrance hole regime where the laser beams interact with nearly $5 \mathrm{~mm}$ of low density $\left(<6 \% n_{c r}\right)$ plasma. Section IV presents quantitative measurements of the effect of polarization smoothing on both SBS and SRS intensity thresholds. The results are summarized in Section VII.

\section{EXPERIMENTAL SETUP}

\section{A. Target Platform}

\section{Standard Low-Density Design}

A standard design for gas-filled cylindrical cylinders ("hohlraums") has been developed over several years $[6$, 7] for use at the OMEGA Laser Facility to study laserplasma instabilities [8-11]. A $351 \mathrm{~nm}$ interaction beam propagates along the hohlraum axis and therefore interact with a long-scale under-dense plasma. This geometry allows the measurements of both the backscattered and forward scattered light and provides a complete accounting of the energy in the interaction beam $[8,9]$.

The standard design uses a $L=2-\mathrm{mm}$ long hohlraum with a diameter of $2 r=1.6 \mathrm{~mm}$ and $0.8 \mathrm{~mm}$ laser entrance holes. The hohlraums are heated by 33-351-nm laser beams $(14.5 \mathrm{~kJ})$ in 3 cones with polar angles of approximately 20, 40 and 62 degrees. The heater beams are pointed at the laser entrance holes and defocused such that their intensities remain below the thresholds for laser-plasma instabilities $\left(\mathrm{I} \sim 10^{14} \mathrm{~W}-\mathrm{cm}^{-3}\right)$. The laser beams primarily heat the inner walls of the gold holhraum, which in turn heat the internal gas via electron conduction. This design has been used to study laserplasma interaction at high temperatures $\left(T_{e}=2-3 \mathrm{keV}\right)$ and low densities $\left(n_{e}=5-10 \% n_{c r}\right)$. At such conditions, stimulated Brillouin scattering (SBS) dominates the interactions, while stimulated Raman scattering (SRS) remains energetically insignificant [8]. Previous studies have used Thomson scattering to characterize the bulk hydrodynamics in this standard low-density platform along the interaction beam path $[12,13]$. The peak electron temperature was measured to be $T_{e}=3.5 \mathrm{keV}$ and at these temperatures, filimentation was successfully mitigated to allow for unambiguous studies of the stimulated Brillouin scattering instability.

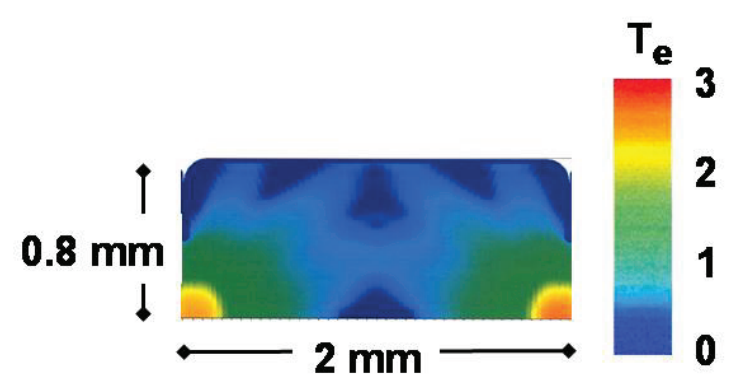

FIG. 2: Simulated electron temperature profile is shown for the conditions $0.1 \mathrm{~ns}$ after the rise of the main heater beams. The "standard" low density target was simulated with an initial high density $\left(12 \% n_{c r}\right)$ gas fill to demonstrate the laser beam imprint evident at early times. This imprint is significantly reduced both at low densities $\left(6 \% n_{c r}\right)$ and in the modified high density target design.

\section{High-Density Design}

In order to study SRS, particularly at plasma conditions relevant to indirect drive fusion ignition experiments, the standard platform was modified to allow for higher density experiments. Previous attempts to study SRS without modifying the standard low-density hohlraum design have produced inconsistent results [14].

Radiation-hydrodynamic simulations with HYDRA [15] have been used to design the laser heated gas-filled hohlraums discussed above and to obtain predictions for the plasma conditions $\left(T_{e}, T_{i}, n_{e}\right.$, etc). Coupled with a linear gain model (LIP [16]), the time and wavelength dependent gains for SRS and SBS are calculated to further guide the experiments and to interpret the measured spectra [7]. The linear gain model uses an average laser beam intensity and by postprocessing the plasma properties from the hydrodynamic simulations calculates the steady-state convective spatial growth rate for SRS and SBS as a function of the scattered light frequency using a kinetic description of the plasma susceptibilities. At each time, the total gain is determined by integrating the growth rate along light rays taking into account the spatially varying plasma conditions. The gain calculations use an averaged laser beam intensity and plasma conditions to evaluate the backscatter from target designs and, as this paper shows, can be a reasonable metric for understanding how the backscatter instabilities will scale in a particular experiment. However, the ability to predict the backscatter reflectivities often relies on the details of the laser beam. For example, the speckles generated by continues phase plates and modified by polarization smoothing and smoothing by spectral dispersion all effect the backscatter and are not accounted for in the gain calculations.

When such simulations are applied to the standard design with a higher gas density, the predicted density along the path of the interaction beam is not uniform. Figure 2 

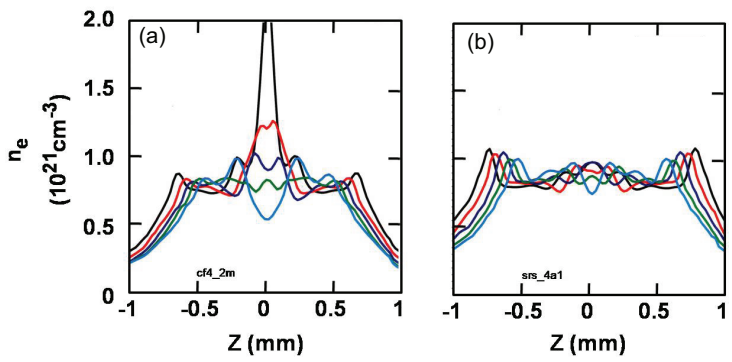

FIG. 3: The axial density profiles are shown for the (a) standard low density design with a high density gas fill and (b) the modified high density design at various times (green) 0.7 ns, (blue) $0.8 \mathrm{~ns}$, (black) $0.9 \mathrm{~ns}$, (red) $1.0 \mathrm{~ns}$, (black) $1.1 \mathrm{~ns}$.

shows a snapshot of the electron temperature for a standard $\mathrm{C}_{5} \mathrm{H}_{12}$ filled hohlraum with initial electron density (when fully ionized) of $12 \% n_{c}$. At such a high density, direct laser heating of the gas becomes important, as shown in Fig. 2 by the early time high-temperature channels created along with the laser beams. Although these high-temperature regions dissipate with time via electron conduction, they leave their mark on the plasma conditions by creating pressure waves, which show up later as density variations (Fig. 3(a)). These density variations lead to large variations in the wavelength dependent gain, which complicate the study of the laser-plasma instabilities. Simulations of lower-density hohlraums used (e.g. $\left.6 \% n_{c}\right)$ do not show such large density variations.

In order to achieve a more uniform high-density plasma, we have redesigned the hohlraum and laser irradiation pattern. The laser beams are focused in front of the laser entrance holes and re-pointed to more uniformly irradiate the hohlraum gas near the axis. Furthermore, elliptical phase plates with a $200 \mu \mathrm{m} \times 300 \mu \mathrm{m}$ FWHM are added to the heater beams. To accommodate these changes, we increased the laser entrance hole diameter from $0.8 \mu \mathrm{m}$ to $1.2 \mu \mathrm{m}$. Two heater beams are used to blow down the windows, 0.5 ns prior to the rise of the main heater beams. Figure 4 shows that the new design has more uniform irradiation patter and Figure 3(b) shows that the on-axis density profiles in the new design have a significant reduction in the level of density variations. This modified design results in relatively small variations in the linear gains for SRS allowing for a detailed study of the laser-plasma interactions to be performed at high densities.

\section{Long High-Temperature Design}

To extend the interaction length, the standard hohlraum design was increased from $2 \mathrm{~mm}$ to $3.5 \mathrm{~mm}$ and $5 \mathrm{~mm}$. An additional 17 laser beams were added through three equally spaced $1200 \mu \mathrm{m}$ diameter laser entrance holes around the equator of the hohlraum. To maintain high temperatures as the hohlraum is lengthened, the
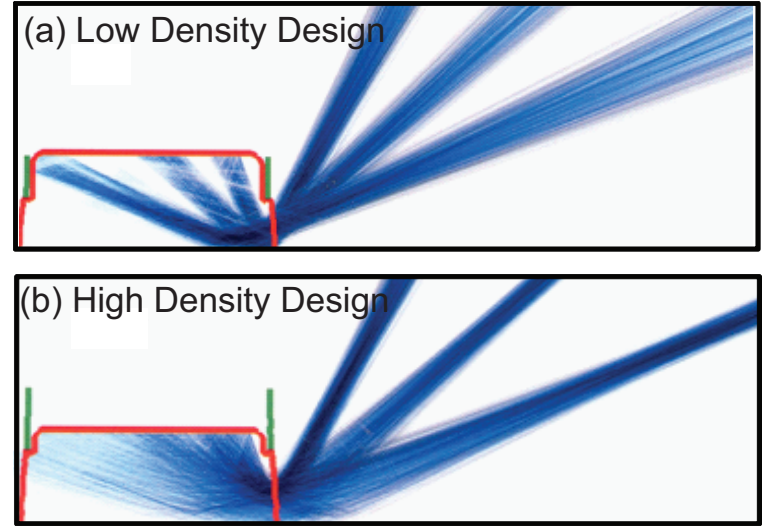

FIG. 4: The laser beam pointing for the (a) standard low density design and (b) the new high density are shown.

total laser heater beam power must be increased. The hydrodynamic simulations exhibit a large region of gas and blow off plasma inside the hohlraum that is nearly isothermal. The temperature of the isothermal region is controlled by a balance of laser heating with increasing thermal energy, radiation cooling, and electron heat transfer to the cold gold walls;

$$
T_{e} \propto\left(n_{e} r\right)^{-2 / 3}\left(\frac{P_{L}}{L}\right)^{2 / 3}
$$

where $P_{L}$ is the total laser power. Therefore maintaining a constant laser power per unit length keeps the temperature approximately constant as the length is increased. This analytical scaling was verified by hydrodynamic simulations $\left(T_{e} \propto\left(\frac{P_{L}}{L}\right)^{0.62}\right)$ where a flux limiter of $f=0.05$ was used and the $P_{L} / L$ was scaled from 4.5 to 9 . The addition of $8.5 \mathrm{~kJ}$ of heater beam energy allowed the total laser power per unit length to be roughly constant between the $2 \mathrm{~mm}$ and $3.5 \mathrm{~mm}$ targets; this produces hot $T_{e}>2.5 \mathrm{keV}$ plasmas for all three target platforms.

\section{B. Interaction Beam}

The $3 \omega$ interaction beam ( $1 \mathrm{~ns}$ square pulse) is focused along the hohlraum axis by a $f / 6.7$ lens to a best vacuum focus at the center of the hohlraum and is timed to arrive $0.3 \mathrm{~ns}$ after the rise of the main heater beams $(0.8$ ns). Two continuous phase plates [17] have been used for these studies to allow the intensity in these studies to range over several orders of magnitude. The high intensity phase plate has a vacuum focus diameter of $100 \mu \mathrm{m}$ FWHM and provides an average intensity at best focus $I_{100 \mu m}=E[J] \times 10^{13}$, where $E$ is the incident laser-beam energy ranging from 100 to $400 \mathrm{~J}$. The low intensity phase plate has a vacuum focus diameter of $200 \mu \mathrm{m}$ FWHM and provides a $1 / 4$ of the intensity of the $100 \mu \mathrm{m}$ beam. 


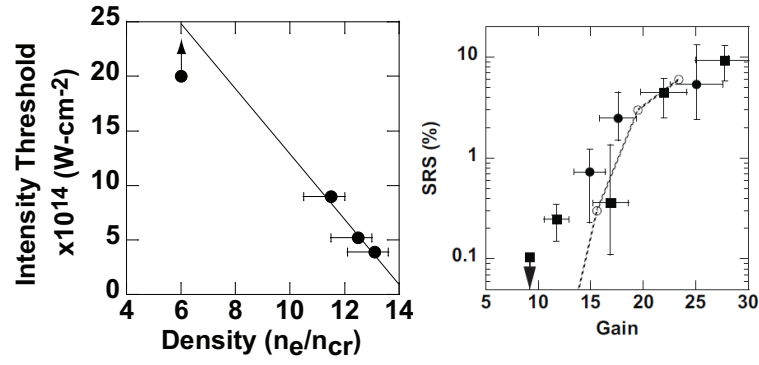

FIG. 5: (a) The intensity at which the SRS reaches 5\% (intensity threshold) is plotted as a function of the plasma density (circles). (b) The measured instantaneous SRS is plotted as a function of the linear gain exponent for intensities below $1 \times 10^{15} \mathrm{~W}-\mathrm{cm}^{-2}$ and a range of densities. The pf3D simulations (open circles) reproduce the measured scaling. Polarization smoothing was used on these shots.

\section{Diagnostics}

Light scattered from the interaction beam is measured using a full-aperture backscatter station (FABS) [18], a near backscatter imager (NBI) [19], and a $3 \omega$ transmit-

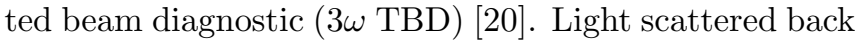
into the original beam cone is collected by the FABS, where the SBS and the SRS spectra and energies are measured. Light backscattered outside of the beam cone is diffusively reflected from a plate surrounding the interaction beam. The plate is imaged onto two charge coupled devices (CCD) which time integrate the SBS and SRS signals. The NBI and FABS are calibrated in both wavelength ranges using a pulsed-calibration system that directs 5-ns laser pulses with known energies from target chamber center [19]. Using this calibration system, the error in the measurements of the SBS power is $10 \%$ and the SRS power is $20 \%$. The $3 \omega \mathrm{TBD}$ allows us to accurately measure the light propagating through the target up to twice the original beam cone. The transmitted energy, spectrum, and temporal beam spray are measured. By correlating the plasma parameters, backscatter, and transmission measurements we are able to account for all the scattered energy from the interaction beam.

\section{HIGH-DENSITY REGIME}

Figure 5 shows that the intensity threshold (intensity at which the SRS backscatter reaches 5\%) has a strong dependence on the plasma density. The electron density was scaled in the 2-mm long target platfrom from $5 \%$ to $13 \% n_{c r}$ while maintaining the electron temperature above $2.5 \mathrm{keV}$ to mitigate filimenation. For conditions below $10 \% n_{c r}$, SRS remained below $<1 \%$ and the backscatter is dominated by SBS (see Sec. V). For densities above $10 \% n_{c r}$, SBS becomes small $<1 \%$ and a $20 \%$ increase in density results in an order of magnitude increase in SRS [21].
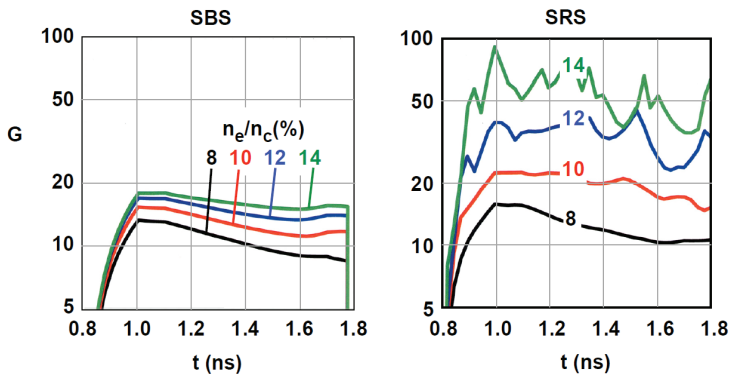

FIG. 6: (a) SBS and (b) SRS linear gain exponents are plotted as a function of time for various densities (black) $8 \% n_{c r}$, (red) $10 \% n_{c r}$, (blue) $12 \% n_{c r}$, and (green) $14 \% n_{c r}$.

Our experimental findings are consistent with theoretical predictions for a uniform plasma. Linear theory predicts that the gain exponent for SRS scales strongly with the background plasma density through the Landau damping and is linear with respect to laser intensity. For the experiments discussed here the linear gain exponent can be written as

$$
G_{S R S} \simeq 0.2\left(\frac{\mathrm{I}}{10^{15}\left[\mathrm{~W}-\mathrm{cm}^{-2}\right]}\right)\left(\frac{1}{\nu_{e} / \omega_{p}}\right) ;
$$

$\nu_{e} / \omega_{p}=\sqrt{\pi / 8}\left(k \lambda_{d}\right)^{-3} \exp \left[-\left(\sqrt{2} k \lambda_{d}\right)^{-2}\right]$ is the normalized Landau damping rate where $k \lambda_{d}$ is the ratio of the Debye length over the wavelength of the plasma wave, and $\omega_{p}$ is the plasma frequency. The normalized damping rate increases from $\nu_{e} / \omega_{p}=0.01$ at $n_{e}=n_{c}=13 \%$ to $\nu_{e} / \omega_{p}=0.035$ at $n_{e} / n_{c}=11 \%$.

Figure 6 shows the time dependent SRS and SBS linear gains for a range of densities calculated by LIP. Simulations with electron heat flux ranging from 0.05 to 0.2 produce a variation in the SRS gain of $10 \%$. Table III summarizes this uncertainty in the calculated SRS gains determined by the uncertainty in heat transport models and uncertainty in absolute density $\left(<0.5 \% n_{c r}\right)$.

\begin{tabular}{|c|c|c|}
\hline$n_{e} / n_{c r}$ & flux limiter & $G_{S R S}$ \\
\hline $10.5 \%$ & 0.05 & 21.8 \\
\hline $11.0 \%$ & 0.05 & 24.3 \\
\hline $11.0 \%$ & 0.10 & 27.9 \\
\hline $11.0 \%$ & 0.20 & 27.2 \\
\hline $11.5 \%$ & 0.05 & 27.2 \\
\hline & \multicolumn{2}{|c}{} \\
\hline
\end{tabular}

Our SRS measurements agree with linear damping rates over a wide range of laser intensities and plasma densities. Figure 5(b) shows that the measured SRS reflectivity scales with linear gain exponent over a range of densities $\left(10.8 \%<n_{e} / n_{c}<13 \%\right)$ and intensities $\left(2.5 \times 10^{14} \mathrm{~W}-\mathrm{cm}^{-2}<I<9 \times 10^{14} \mathrm{~W}-\mathrm{cm}^{-2}\right)$. The instantaneous backscatter is determined at a time 0.7 ns after the rise of the heater beams $(1.2 \mathrm{~ns})$ once the plasma reaches high plasma temperatures where filamentation effects are negligible. The instantaneous reflectivity is calculated by averaging over a 100 ps range and the error bars are given by the extreme reflectivities 


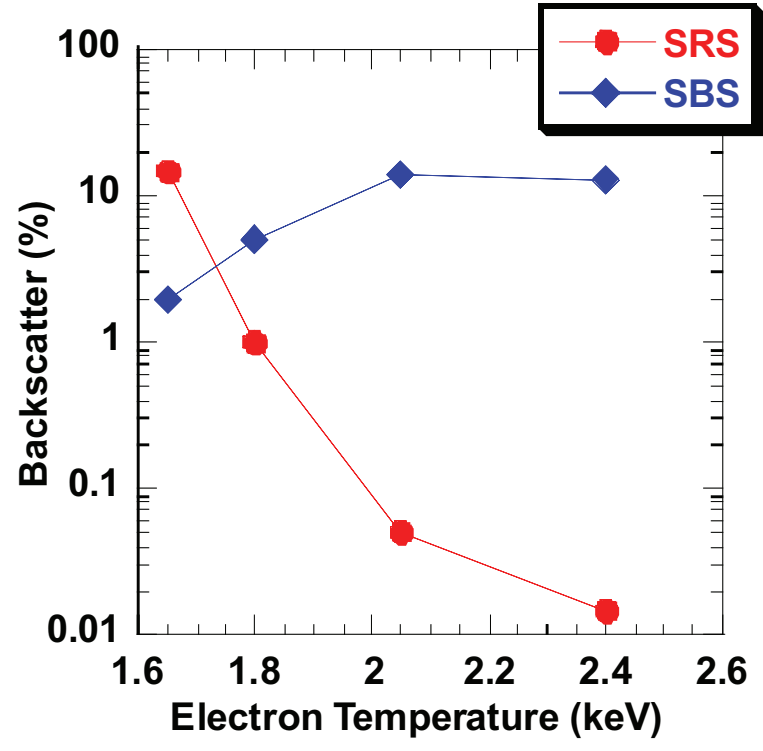

FIG. 7: The (circles) SRS and (diamonds) SBS backscatter is plotted as a function of the electron temperature for an intensity of $10^{15} \mathrm{~W}-\mathrm{cm}^{-2}$.

within this range. Furthermore, Fig. 5(b) demonstrates that the resulting increase in scattering resulting from the increased density can be mitigated by reducing the laser beam intensity to maintain a constant linear gain exponent. Indeed, the density thresholds measured in Fig. 5(a) correspond to the nearly the same SRS linear gain $\left(G_{13 \%}=22, G_{11 \%}=24\right)$.

These results highlight the sensitivity of SRS to density and indicate the importance of controlling the plasma density and the laser beam intensity in targets where SRS will can have an adverse effect. Current ignition designs for the NIF mitigate SRS by remaining in the strongly damped regime $\left(k \lambda_{d}>0.4\right)$ when the laser beam intensity is high and keeping the laser intensity below the backscatter threshold where the density is high and the temperature is low $\left(k \lambda_{d}<0.4\right)$, but future high yield and high gain experiments will likely push these intensity limits and other mitigation techniques will be required.

\section{MIDDLE-DENSITY REGIME}

At plasma densities of $8.5 \% n_{c r}$, the SBS and SRS gains are nearly equivalent and a competition between these instabilities occurs. The peak electron temperature was scaled from $1.5 \mathrm{keV}$ to $2.4 \mathrm{keV}$ by changing the total heater beam energy from $9 \mathrm{~kJ}$ to $14.5 \mathrm{~kJ}$. Figure 7 shows that SBS dominates the backscatter for electron temperatures above $2 \mathrm{keV}$, but at lower temperatures SRS becomes energetically significant reducing the available laser power for driving SBS.
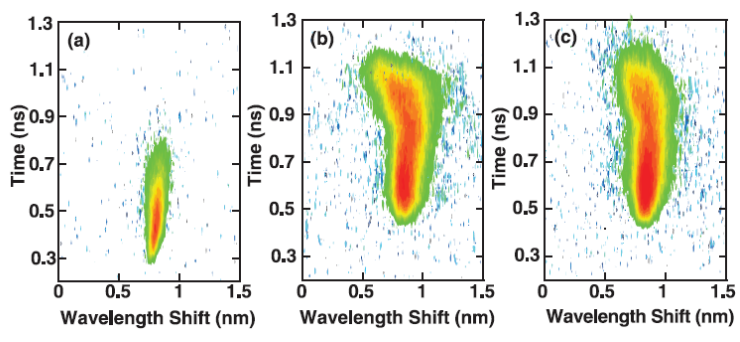

FIG. 8: The SBS spectra is shown for the 2-mm (a), 3.5$\mathrm{mm}$ (b), and 5-mm (c) long targets at a vacuum intensity of $1.2 \times 10^{15} \mathrm{~W}-\mathrm{cm}^{-2}$

\section{LOW-DENSITY REGIME}

At an electron density of $6 \% n_{c r}$, the plasma length was scaled to $5 \mathrm{~mm}$ while maintaining an electron temperature above $2.5 \mathrm{keV}$. Figure 8 shows that the SBS peaks early in time when the plasma is cold and then, in the 2-mm long target, it drops below detection as the plasma temperature increases. For electron temperatures above $3 \mathrm{keV}$, the total backscatter is reduced below $1 \%$ for intensities less than $2.5 \times 10^{15} \mathrm{~W}-\mathrm{cm}^{-2}[8]$. When the plasma length, the SBS remains significant throughout the experiment despite the increasing electron temperature. The spectra confirm that the increased laser beam energy maintained the electron temperature above $2.5 \mathrm{keV}$ by the $>0.8 \mathrm{~nm}$ SBS wavelength shift. In the longer targets, the spectra broaden late in time as a result of the increased ion temperature consistent with previous Thomson scattering ion temperature measurements [6].

For an intensity of $6 \times 10^{14} \mathrm{~W}-\mathrm{cm}^{-2}$ the backscatter increases from $<0.05 \%$ to $>10 \%$ when increasing the plasma length from 1.3 to $4.0 \mathrm{~mm}$. The intensity threshold is measured to be $\mathrm{I}_{5 \mathrm{~mm}}=4 \times 10^{14} \mathrm{~W}-\mathrm{cm}^{-2}$ for propagation through this 5 -mm long target while the threshold is a factor of 2.5 higher in the 2-mm long target $\left(I_{2 m m}=10^{15} \mathrm{~W}-\mathrm{cm}^{-2}\right)[22]$. These experiments demonstrate that backscatter in a 4-mm long plasma (the 5$\mathrm{mm}$ long target produces a $4-\mathrm{mm}$ long plasma) can be controlled at ignition plasma conditions and intensities. The experiments show that the backscatter threshold is a function of the plasma length times the intensity; an increase in plasma length can be compensated for by an equivalent reduction in intensity as suggested by the SBS linear gain

$G_{s b s} \simeq 290 \lambda_{0}[\mu m]\left(\frac{n_{e}}{n_{c r}}\right)\left(\frac{L[m m]}{T_{e}[k e V]}\right)\left(\frac{\omega_{a}}{\nu_{a}}\right) I_{15}\left[\mathrm{~W}-\mathrm{cm}^{-2}\right]$

where the Landau damping rate $\nu_{a}$ normalized to the ionacoustic frequency $\omega_{a}$ for our conditions is $\nu_{a} / \omega_{a} \simeq 0.15$.

Figure 9 shows the measured SBS as a function of the calculated SBS gain over a variety of lengths, intensities, and plasma conditions. Each point is obtained by averaging the measured SBS over a 100 ps range and calculating a SBS gain using LIP for the associated time 


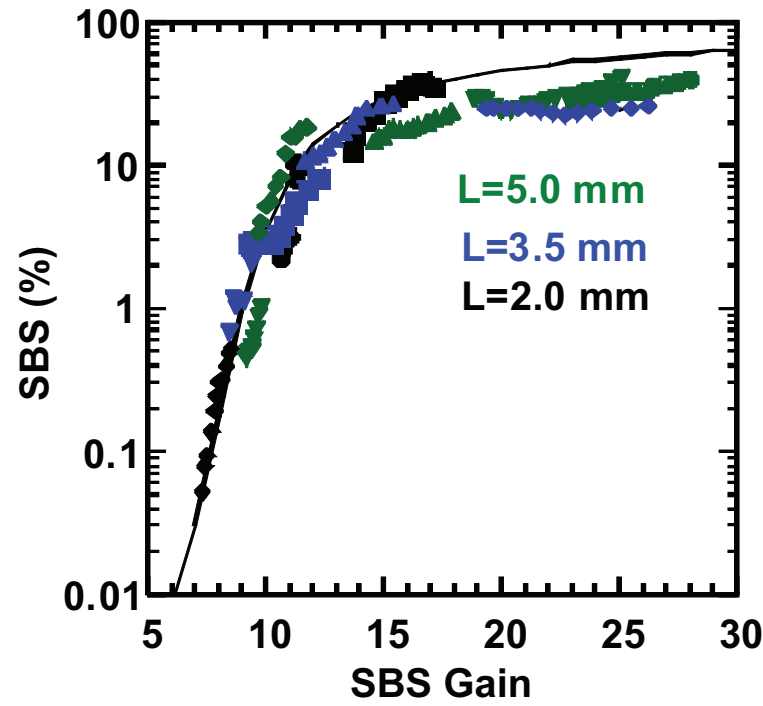

FIG. 9: The measured instantaneous SBS reflectivity is plotted as a function of the linear gain exponent for various intensities (diamonds) $x \times 10^{14} \mathrm{~W}-\mathrm{cm}^{-2}$, (down-triangles) $x \times 10^{14}{\mathrm{~W}-\mathrm{cm}^{-2}}$, (triangles) $x \times 10^{14} \mathrm{~W}-\mathrm{cm}^{-2}$ and target lengths, (black) $2 \mathrm{~mm}$, (blue) $3.5 \mathrm{~mm}$, (green) $5 \mathrm{~mm}$.

between $0.6 \mathrm{~ns}$ and $1.1 \mathrm{~ns}$ after the rise of the heater beams. This shows that linear gain is a good metric to describe backscatter at these conditions.

\section{POLARIZATION SMOOTHING}

Laser plasma instabilities are sensitive to parameters that are not encompassed by the linear gain calculations. Specifically laser beam smoothing techniques [e.g., random phase plates (RPP), smoothing by spectral dispersion (SSD), polarization smoothing(PS)] are not directly accounted for in the calculation of the linear gains. For example, the reduction in intensity contrast and the longitudinal randomization of the polarization that occurs when applying polarization smoothing does not change the average laser beam intensity and therefore the calculated gains. A more sophisticated model, such as pf3D, which accounts for speckles and polarization effects is needed to calculate these effects.

Figure 10 shows that applying polarization smoothing reduces the threshold for both SBS and SRS. At low densities, $6 \% n_{c r}$, where SRS remains below $1 \%$, polarization smoothing reduces the SBS from $5 \%$ to less than $0.1 \%$ at an intensity of $1.3 \times 10^{15} \mathrm{~W}-\mathrm{cm}^{-2}$. Applying polarization smoothing allows the intensity to be increased by a factor of 1.7 before the SBS exceeds the initial $5 \%$. This increased threshold in long-scale length plasmas is attributed to the longitudinal mixing of the polarization, not a reduction in contrast [23].

Figure 10(b) shows that polarization smoothing also reduces the threshold for SRS at higher densities. At a density of $11.5 \% n_{c r}$, SBS remains below $1 \%$, and SRS
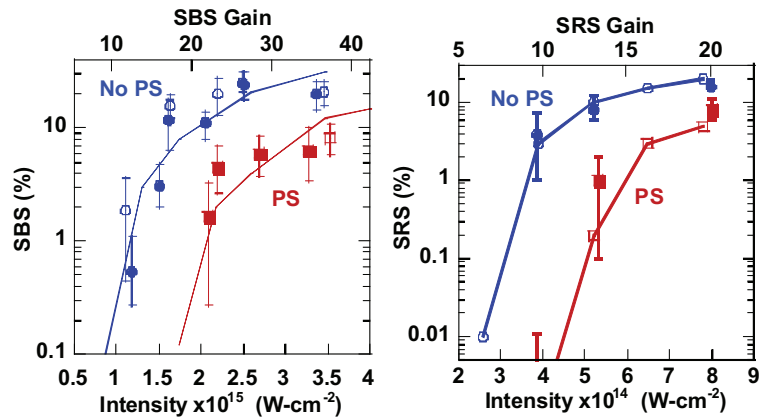

FIG. 10: The instantaneous SBS (a) and SRS (b) 700 ps after the rise of the heater beams are plotted as a function of the interaction beam intensity. Two laser-smoothing conditions are shown: CPP-only (blue circles), CPP and PS (red squares). The simulated reflectivies calculated by pF3d are shown (solid curves). The SRS data are for a density of $n_{e} / n_{c r}=11.5 \%$ and the SBS data are for a density of $n_{e} / n_{c r}=6 \%$.

becomes the dominant scattering process (see Sec. III). Now adding polarization smoothing increases the SRS intensity threshold by a factor of 1.6. These observations shows that polarization smoothing is an effective mitigation technique for controlling both SRS and SBS in a high-temperature inertial confinement fusion plasma where filamentation effects are negligible.

Although the linear gains do not directly account for the addition of polarization smoothing, simulations of the interaction beam propagation and the instantaneous SRS reflectivity using the code Pf3d [24] agree well with the measured backscatter for both cases-with and without polarization smoothing. These three-dimensional calculations use a paraxial approximation to model the whole laser beam propagating through the full 2-mm long hohlraum plasma. The code includes models for both SRS and SBS backscattering and shows that using a fluid-based modeling of SRS and SBS including linear kinetic corrections (i.e. Landau damping), coupled to accurate hydrodynamic profiles and a realistic description of the laser intensity pattern generated by various smoothing techniques leads to quantitative agreement between the measurement and calculated relfectivities [25, 26].

\section{SUMMARY}

In summary, we have performed a series of experiments to study laser-plasma interactions in preparation for the National Ignition Campaign. The experiments presented in this manuscript have quantitatively measured the density, temperature, and intensity thresholds for backscatter at conditions directly applicable to indirect drive ignition plasma experiments. The experimental results are compared with linear gain calculations over a wide range of parameters. The complete set of experiments along with the relative success of modeling the absolute backscatter levels has built confidence in our tools used 
to extrapolate to the National Ignition Campaign.

This work performed under the auspices of the U.S.
Department of Energy by Lawrence Livermore National Laboratory under Contract DE-AC52-07NA27344.
[1] E. MOSES and C. Wuest, FUSION SCIENCE AND TECHNOLOGY 47, 314 (2004).

[2] J. Lindl, P. Amendt, R. Berger, S. Glendinning, S. Glenzer, S. Haan, R. Kauffman, O. Landen, and L. Suter, PHYSICS OF PLASMAS 11, 339 (2004).

[3] J. Fernandez, J. Cobble, D. Montgomery, M. Wilke, and B. Afeyan, PHYSICS OF PLASMAS 7, 3743 (2000).

[4] B. MacGowen, B. Afeyan, C. Back, R. Berger, G. Bonnaud, M. Casanova, B. Cohen, D. Desenne, D. DuBois, A. Dulieu, et al., PHYSICS OF PLASMAS 3, 2029 (1996).

[5] T. Boehly, D. Brown, R. Craxton, R. Keck, J. Knauer, J. Kelly, T. Kessler, S. Kumpan, S. Loucks, S. Letzring, et al., OPTICS COMMUNICATIONS 133, 495 (1997).

[6] D. Froula, J. Ross, L. Divol, N. Meezan, A. MacKinnon, R. Wallace, and S. Glenzer, PHYSICS OF PLASMAS 13, (2006).

[7] N. Meezan, R. Berger, L. Divol, D. Froula, D. Hinkel, O. Jones, R. London, J. Moody, M. Marinak, C. Niemann, et al., PHYSICS OF PLASMAS 14, (2007).

[8] D. Froula, L. Divol, N. Meezan, S. Dixit, J. Moody, P. Neumayer, B. Pollock, J. Ross, and S. Glenzer, PHYSICAL REVIEW LETTERS 98, (2007).

[9] C. Niemann, R. Berger, L. Divol, D. Froula, O. Jones, R. Kirkwood, N. Meezan, J. Moody, J. Ross, C. Sorce, et al., PHYSICAL REVIEW LETTERS 100, (2008).

[10] P. Neumayer, R. Berger, L. Divol, D. Froula, R. London, B. MacGowan, N. Meezan, J. Ross, C. Sorce, L. Suter, et al., PHYSICAL REVIEW LETTERS 100, (2008).

[11] P. Neumayer, R. Berger, D. Callahan, L. Divol, D. Froula, R. London, B. MacGowan, N. Meezan, P. Michel, J. Ross, et al., PHYSICS OF PLASMAS 15, (2008).

[12] J. Ross, D. Froula, A. Mackinnon, C. Sorce, N. Meezan, S. Glenzer, W. Armstrong, R. Bahr, R. Huff, and K. Thorp, REVIEW OF SCIENTIFIC INSTRUMENTS 77, (2006).

[13] D. Froula, J. Ross, L. Divol, and S. Glenzer, REVIEW OF SCIENTIFIC INSTRUMENTS 77, (2006).

[14] J. Kline, D. Montgomery, H. Rose, S. Goldman, D. Froula, J. Ross, R. Stevenson, and P. Lushnikov, in Journal of Physics (2008), vol. 112 of IFSA200\%, p. 022030 .

[15] M. Marinak, G. Kerbel, N. Gentile, O. Jones, D. Munro, S. Pollaine, T. Dittrich, and S. Haan, PHYSICS OF PLASMAS 8, 2275 (2001)

[16] E. Williams (1998).

[17] S. DIXIT, I. THOMAS, B. WOODS, A. MORGAN, M. HENESIAN, P. WEGNER, and H. POWELL, AppLIED OPTICS 32, 2543 (2003).

[18] S. Regan, D. Bradley, A. Chirokikh, R. Craxton, D. Meyerhofer, W. Seka, R. Short, A. Simon, R. Town, B. Yaakobi, et al., PHYSICS OF PLASMAS 6, 2072 (1999).

[19] P. Neumayer, C. Sorce, D. Froula, L. Divol, V. Rekow, K. Loughman, R. Knight, S. Glenzer, R. Bahr, and W. Seka, REVIEW OF SCIENTIFIC INSTRUMENTS 79, (2008).

[20] D. Froula, V. Rekow, C. Sorce, K. Piston, R. Knight, S. Alvarez, R. Griffith, D. Hargrove, J. Ross, S. Dixit, et al., REVIEW OF SCIENTIFIC INSTRUMENTS 77, (2006).

[21] D. Froula, L. Divol, P. Davis, J. Palastro, P. Michel, V. Leurent, S. Glenzer, B. Pollock, and G. Tynan, PLASMA PHYSICS AND CONTROLLED FUSION 51, (2009).

[22] D. Froula, L. Divol, R. London, P. Michel, R. Berger, N. Meezan, P. Neumayer, J. Ross, R. Wallace, and S. Glenzer, PHYSICAL REVIEW LETTERS 100, (2008).

[23] D. Froula, L. Divol, R. Berger, R. London, N. Meezan, D. Strozzi, P. Neumayer, J. Ross, S. Stagnitto, L. Suter, et al., PHYSICAL REVIEW LETTERS 101, (2008).

[24] R. Berger, C. Still, E. Williams, and A. Langdon, PHYSICS OF PLASMAS 5, 4337 (1998).

[25] L. Divol, R. Berger, N. Meezan, D. Froula, S. Dixit, P. Michel, R. London, D. Strozzi, J. Ross, E. Williams, et al., PHYSICS OF PLASMAS 15, (2008).

[26] L. Divol, R. Berger, N. Meezan, D. Froula, S. Dixit, L. Suter, and S. Glenzer, PHYSICAL REVIEW LETTERS 100, (2008). 